\title{
Triaging Patients Undergoing Endoscopy During COVID-19 Pandemic
}

\author{
Sundeep Lakhtakia' $\quad$ Mohan Ramchandani ${ }^{1}$ \\ ${ }^{1}$ Asian Institute of Gastroenterology, AIG Hospitals, Hyderabad, \\ India
}

\begin{abstract}
Address for correspondence Sundeep Lakhtakia, MD DM MNAMS FASGE, Director Endoscopy \& EUS, Asian Institute of Gastroenterology, AIG Hospitals, Gachibowli, Hyderabad, Telangana 500032, India

(e-mail: drsundeeplakhtakia@gmail.com).
\end{abstract}

\begin{abstract}
Keywords

- Key words Covid-19 pandemic

- triage

- endoscopy

- screening

"Triaging for endoscopy" is the process to determine the most deserving patients after careful scrutiny amongst a pool of patients who report for Gastrointestinal (GI) Endoscopy diagnostic or interventional procedures that include Esophago-gastro-duodenoscopy (OGD), Colonoscopy, ERCP, EUS and Enteroscopy. The purpose of triaging at endoscopy is to prevent transmission of SARS-COV2 infection from suspected patient to the health care workers involved at endoscopy. The three professional Indian Gastroenterology bodies namely Society of Gastrointestinal Endoscopy of India (SGEI), Indian Society of Gastroenterology (ISG) \& Indian Association for the study of the liver (INASL) have recently provided guidance of scheduling of endoscopic procedures in the current Covid-9 pandemic. Similar suggestions have been promulgated by other international bodies.
\end{abstract}

"Triaging for endoscopy" is the process to determine the most deserving patients after careful scrutiny among a pool of patients who report for gastrointestinal (GI) endoscopy diagnostic or interventional procedures that include esophago-gastro-duodenoscopy (OGD), colonoscopy, endoscopic retrograde cholangio-pancreatography (ERCP), endoscopic ultrasound (EUS), and enteroscopy. ${ }^{1,2}$

The three professional Indian Gastroenterology bodies, namely, Society of Gastrointestinal Endoscopy of India (SGEI), Indian Society of Gastroenterology (ISG) \& Indian Association for the study of the liver (INASL) have recently provided guidance for scheduling of endoscopic procedures in the current COVID-19 pandemic. $^{3}$ Similar suggestions have been promulgated by other international bodies. ${ }^{4}$

\section{Why is Triaging Important in the Current COVID-19 Pandemic?}

In preCOVID-19 days, all patients scheduled for endoscopy procedures underwent them at a scheduled time using standard personal protection equipment (PPE). This situation has changed drastically since the SARS-CoV-2 virus related COVID-19 pandemic that is highly contagious and has the potential to spread by aerosols and feco-oral route and can persist for prolonged period on different surfaces. ${ }^{3,5,6}$ In the current scenario, like so many other modifications in our lifestyle, social behavior and practices, the current pandemic has also completely changed the way endoscopy practices are being considered and performed.

1. Reduce risk of transmission. The main purpose of triaging is to reduce the risk of transmission to healthcare personnel who are exposed to saliva, expectorations, vomitus, and feces. A large proportion (up to $80 \%$ ) of population can be asymptomatic carriers of the COVID-19 contagious virus, who may also include the potential subjects scheduled for endoscopy, and who can then spread to theatre staff during the aerosol-generating endoscopy procedures through saliva, expectorations and feces.

2. PPE requirement. Appropriate PPE are required by all endoscopy staff and patients to prevent to-and-fro transmission. Triaging helps to avoid current or anticipated shortage of PPE (N95 mask, gloves, gowns, etc.) for medical personnel taking care of patients, with or without confirmed COVID-19. Unfortunately, the scarcity of PPE has been observed in many countries, including the developed nations, due to limited inventory, supply chain issues, and failed anticipation of the epidemic. The expenses and other efforts involved to ensure adequate personal protection to all involved are associated with enormous cost burden to the unit and eventually to the entire healthcare system. To match the "supply and demand," it is logical to
License terms

()(1) $\Theta \circledast$ 
select the most deserving patients for endoscopy procedures where it is going to make the maximum difference in terms of immediate outcome.

3. Testing. Ideally, all patients due for endoscopy procedures require testing to confirm or exclude active viral infection by polymerase chain reaction (PCR). Due to current limited supply of available testing kits along with the cost involved (Rs 4500 set as the minimum cost recommended by Government of India), wearing of appropriate PPE turns out to be beneficial both in terms of cost and safety.

4. Resource allocation. Allocate human resources (nurses, technicians and other staff) to most deserving patients. ${ }^{7}$ It is anticipated that an increasing number of procedures will be required in patients known to be COVID-19 positive in future. Hence, dedicated endoscopy units having a separate entrance, exclusive endoscopes designated to specific endoscopy room, and subsequently reprocessed in a dedicated adjacent reprocessing room, thus creating an "infected pathway" separated by "noninfected" areas to minimize contamination, may be required. It may also be appropriate that only selected members of the team are specifically dedicated to this pathway after proper training on the use of PPE. ${ }^{8}$

\section{How to Triage?}

\section{Scheduling Endoscopic Procedures}

All scheduled endoscopy procedures should be divided into three categories based on their urgency as follows:

A. Emergency endoscopy: includes life-saving measures and needs to be done on emergency basis, for example, acute upper/lower GI bleeding, removal of impacted foreign body, cholangitis, and management of GI perforations., ${ }^{3,4}$

B. Urgent endoscopy: includes procedures that the treating clinician feels will significantly change the management and clinical outcome in a short period of time. Urgent endoscopy procedures can be performed on a case-to-case basis. Procedures included drainage of infected pancreatic fluid collection, diagnosis and staging of GI cancers, draining of malignant biliary obstruction, management of highgrade dysplasia (HGD) or early intramucosal cancer in the esophagus, stomach or large colonic polyps at high-risk of submucosal invasion, nutritional support by nasojejunal (NJ) tube/percutaneous endoscopic gastrostomy (PEG) placement, stenting for malignant luminal obstruction (growth in the esophagus, colon and duodenum), upper GI bleeding without hemodynamic instability, colonoscopy for melena after negative upper GI endoscopy, severe anemia with no hemodynamic instability, foreign body in the stomach, lymph node EUS sampling, benign stricture requiring dilation/stenting, and necrosectomy. ${ }^{3,4}$

C. Routine endoscopy: Those who do not fall in either of the above two categories, for example, all routine referrals for endoscopy procedures (dyspepsia without alarm symptoms, gastroesophageal reflux disease [GERD], irritable bowel syndrome [IBS]), screening and surveillance endoscopy (for Barrett's esophagus, inflammatory bowel syndrome [IBD], postendoscopy resection), and bariatric
GI endoscopy procedures (e.g., intragastric balloons and endoscopic sleeve gastroplasty).

Routine endoscopy procedures can be postponed for a period till the risk of transmission at endoscopy of virus substantially reduces or unless patients develop significant worsening of symptoms in the intervening period.

\section{Workforce Modifications}

It is appropriate to create a working team and workflow pattern that provide a clear job description with backup plans. The workflow should be separate to minimize cross-contamination by having $>1$ team with rotational posting at predefined intervals (such as weekly). One team is o-site and providing direct clinical care. The other team/s coordinates the clinical care remotely, thus minimizing the risk of exposure and providing backup coverage if needed. ${ }^{3,5}$

\section{Screening at Endoscopy}

Risk stratification of patients for possible COVID-19 infection should be started at the time of scheduling endoscopy (-Fig. 1). This should be followed-up at least 1 day prior to GI endoscopy (preferably by phone) and then again on the day of procedure by enquiring for symptoms and contacts; or if/ when available through tests for virus infection or immunity. ${ }^{2,3}$

\section{Pre-Endoscopy Procedure Screening}

Patients scheduled for GI endoscopy procedures should be categorized according to their risk of harbouring SARS-CoV-2 infection into three categories as follows: ${ }^{3,9}$

Low-risk:

- No symptoms (cough, fever, breathlessness, and diarrhea).

- No stay (in high-risk areas* in the last 14 days)

- No contact (with a COVID-19 patient)

Intermediate-risk:

- Symptoms present BUT

- No stay (in high-risk areas* in the last 14 days) AND

- No contact (with a COVID-19 patient)

or

- No symptoms BUT

- Contact with known COVID-19 patient OR

- Stay in high-risk area in the last 14 days

High-risk:

- At least one symptom present AND

- Contact with COVID-19 patient OR

- Stay in a high-risk area

NB. * "high-risk" areas are all areas where more than 1000 cases have been confirmed till date; this is changing on a daily basis.

- All emergency procedures should be considered "highrisk" if patient history cannot be adequately assessed

\section{Endoscopy}

- Routine temperature check of all patients scheduled for endoscopy 


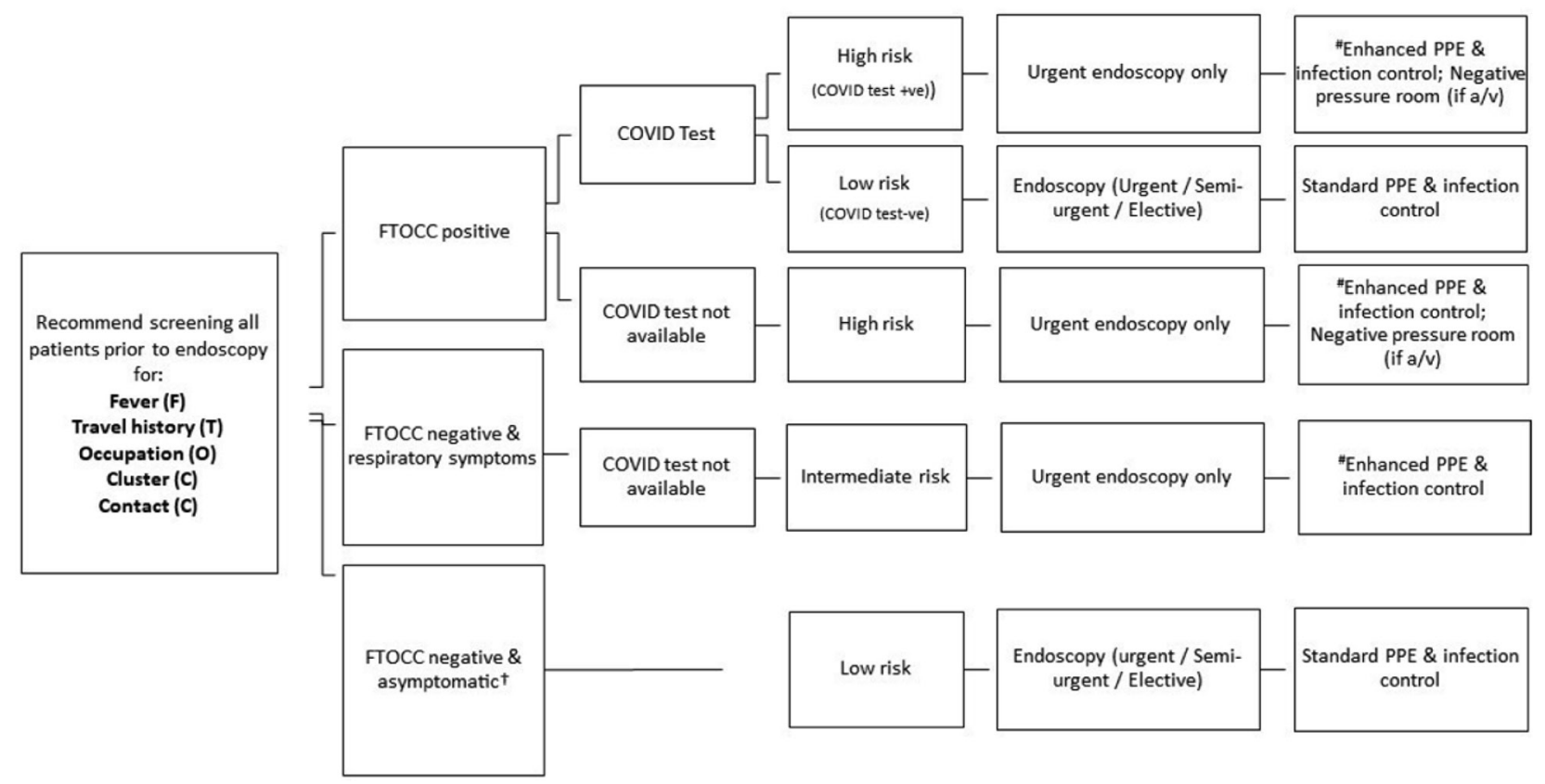

Fig. 1 Flowchart to prescreen and triage patients for endoscopy during COVID-19 outbreak. ${ }^{9}$

- Postpone high-risk procedures for reasonable time till the treat perception of COVID-19 is over, except in case of emergency. In case of high-risk exposure or symptoms, follow the recommended protocol provided by ministry of health and family welfare (MOHFW), Government of India.

\section{Procedure Room}

- Restrict staff in the endoscopy area to the minimum required during the procedure.

- Appropriate PPE should be worn by all members of the endoscopy team on the basis of risk stratification. ${ }^{3}$

- Adequate hand washing before and after handling patients must be emphasized for all healthcare workers (HCWs).

- Endoscopic accessories are preferably restricted to disposables.

- Minimum furniture in the room.

- Restrict the use of gadgets/computer to single operator for data entry, communication, etc.

The Indian GI societies (SGEI, ISG, INASL) recommend all endoscopists to consider only emergency and urgent endoscopy procedures till the current threat due to COVID-19 is significantly reduced or gets over. Routine endoscopy procedures can be postponed till the current risk reduces or if there is a change in the risk category in the intervening period.

\section{Conflict of Interest}

None declared.

\section{References}

1 Philip M, Lakhtakia S, Aggarwal R, Madan K, Saraswat V, Makharia G. Joint Guidance from SGEI, ISG and INASL for gastroenterologists and gastrointestinal endoscopists on the prevention, care and management of patients with COVID-19. J Clin Exp Hepatol 2020;(e-pub ahead of print). doi https://doi. org/10.1016/j.jceh.2020.04.001
2 Repici A, Maselli R, Colombo M, et al. Coronavirus (COVID19) outbreak: what the department of endoscopy should know. Gastrointest Endosc 2020;(e-pub ahead of print). doi 10.1016/j.gie.2020.03.019

3 Philip M, Lakhtakia S, Aggarwal R, Madan K, Saraswat V, Makharia G. Joint Guidance from the Society of Gastrointestinal Endoscopy of India (SGEI), Indian Society of Gastroenterology (ISG), and Indian National Association for Study of the Liver (INASL) for Gastroenterologists and Gastrointestinal Endoscopists on the prevention, care, and management of patients with COVID-19. J Dig Endosc 2020;(e-pub ahead of print) Available at http://www.thieme-connect.de/DOI/ DOI?10.1055/s-0040-1709837 Accessed April 24, 2020

4 European Society of Gastrointestinal Endoscopy, European Society of Gastroenterology and Endoscopy Nurses and Associates. ESGE and ESGENA Position Statement on gastrointestinal endoscopy and the COVID-19 pandemic. Endoscopy 2020;(e-pub ahead of print). Available at https://www.esge. com/esge-and-esgena-position-statement-on-gastrointestinal-endoscopy-and-the-covid-19-pandemic/\%0D Accessed April, 24, 2020

5 Soetikno R, Teoh AY, Kaltenbach T, et al. Considerations in performing endoscopy during the COVID-19 pandemic. Gastrointest Endosc 2020;(e-pub ahead of print). doi 10.1016/j .gie.2020.03.3758

6 Gu J, Han B, Wang J. COVID-19: gastrointestinal manifestations and potential fecal-oral transmission. Gastroenterology 2020;(e-pub ahead of print). doi 10.1053/j.gastro.2020.02.054

7 Ponchon T, Chaussade S. COVID-19: How to select patients for endoscopy and how to reschedule the procedures? Endosc Int Open 2020;08:699-700

8 Cortegoso Valdivia P, Le Grazie M, Gaiani F, Decembrino F, De' Angelis GL. Separated pathways in the endoscopy unit for COVID-19 patients. Gastrointest Endosc 2020; (e-pub ahead of print). doi 10.1016/j.gie.2020.03.3841

9 Chiu PWY, Ng SC, Inoue $\mathrm{H}$, et al. Practice of endoscopy during COVID-19 pandemic: position statements of the Asian Pacific Society for Digestive Endoscopy (APSDE-COVID statements) Gut 2020;(e-pub ahead of print). doi 10.1136/ gutjnl-2020-321185 